\title{
BMJ Open Identifying neurocognitive outcomes and cerebral oxygenation in critically ill adults on acute kidney replacement therapy in the intensive care unit: the INCOGNITO-AKI study protocol
}

\author{
Natasha Arianne Jawa (1) , ${ }^{1}$ Rachel M Holden, ${ }^{2}$ Samuel A Silver, ${ }^{2}$ \\ Stephen H Scott, ${ }^{1,3}$ Andrew G Day, ${ }^{4,5}$ Patrick A Norman, ${ }^{5}$ Benjamin Y M Kwan, ${ }^{6}$ \\ David M Maslove, ${ }^{7,8}$ John Muscedere, ${ }^{7,8}$ John Gordon Boyd ${ }^{1,2,7,8}$
}

To cite: Jawa NA, Holden RM, Silver SA, et al. Identifying neurocognitive outcomes and cerebral oxygenation in critically ill adults on acute kidney replacement therapy in the intensive care unit: the INCOGNITO-AKI study protocol. BMJ Open 2021;11:e049250. doi:10.1136/ bmjopen-2021-049250

- Prepublication history for this paper is available online To view these files, please visit the journal online (http://dx.doi. org/10.1136/bmjopen-2021 049250).

Received 19 January 2021 Accepted 02 August 2021

Check for updates

(C) Author(s) (or their employer(s)) 2021. Re-use permitted under CC BY-NC. No commercial re-use. See rights and permissions. Published by BMJ.

For numbered affiliations see end of article.

Correspondence to

Dr John Gordon Boyd;

gordon.boyd@kingstonhsc.ca

\section{ABSTRACT}

Introduction Initiation of acute kidney replacement therapy (KRT) is common in critically ill adults admitted to the intensive care unit (ICU), and associated with increased morbidity and mortality. KRT has been linked to poor neurocognitive outcomes, leading to reduced quality of life and increased utilisation of healthcare resources. Adults on dialysis in the ICU may be particularly at risk of neurocognitive impairment, as survivors of critical illness are already predisposed to developing cerebrovascular disease and cognitive dysfunction long-term relative to healthy controls. Regional cerebral oxygen saturation may provide a critical early marker of long-term neurocognitive impairment in this population. This study aims to understand cerebral oxygenation in patients undergoing KRT (continuous or intermittent) in the ICU. These findings will be correlated with long-term cognitive and functional outcomes, and structural brain pathology.

Methods and analysis 108 patients scheduled to undergo treatment for acute kidney injury with KRT in the Kingston Health Sciences Centre ICU will be recruited into this prospective observational study. Enrolled patients will be assessed with intradialytic cerebral oximetry using near infrared spectroscopy. Delirium will be assessed daily with the Confusion Assessment Method-ICU (CAM-ICU) and severity quantified as cumulative CAM-ICU-7 scores. Neurocognitive impairment will be assessed at 3 and 12 months after hospital discharge using the Kinarm and Repeatable Battery for the Assessment of Neuropsychological Status. Structural brain pathology on MRI will also be measured at the same timepoints. Driving safety, adverse events and medication adherence will be assessed at 12 months to evaluate the impact of neurocognitive impairment on functional outcomes.

Ethics and dissemination This study is approved by the Queen's University Health Sciences/Affiliated Teaching Hospitals Research Ethics Board (DMED-2424-20). Results will be presented at critical care conferences, and a lay summary will be provided to patients in their preferred format.

Trial registration number NCT04722939

\section{Strengths and limitations of this study}

- This will be the first study to use a comprehensive battery of neurological tests to interrogate both short-term and long-term neurological impairment in critically ill adults on dialysis.

- This study will employ robotic technology in a novel patient population to provide a quantitative assessment of neurocognitive function across a range of cognitive domains.

- The targeted sample size will permit statistically powered conclusions to be drawn from primary analyses.

- The study protocol will be conducted at a single centre.

\section{INTRODUCTION}

Due to advances in critical care, the number of individuals surviving critical illness has increased significantly over the past two decades. This increased survivorship has led to recognition of post-intensive care unit (ICU) syndrome, characterised by longterm cognitive, psychological and functional limitations. ${ }^{1}$ For example, 1 year following ICU admission, survivors have cognitive performance similar to individuals with mild dementia or moderate traumatic brain injury. ${ }^{2}$ These impairments have important implications for quality of life, as only approximately two-thirds of previously employed individuals ever return to work following their ICU stay. ${ }^{34}$ The underlying cause of this cognitive impairment after critical illness is unclear, although it is known that delirium is one of the most consistent risk factors for long-term cognitive impairment. ${ }^{5}$

Delirium is an acute change in level of consciousness, characterised by impaired 
attention and disorganised thinking, affecting up to $80 \%$ of critically ill patients. ${ }^{6}$ Our group has demonstrated that low cerebral oxygenation, as measured by near-infrared spectroscopy (NIRS), is an independent risk factor for delirium in critically ill patients. ${ }^{7}$ However, delirium is clearly multifactorial, and other key risk factors have also been described, including acute kidney injury (AKI). ${ }^{8}$

The incidence of severe AKI is on the rise, with up to $13 \%$ of adults admitted to ICUs per year requiring kidney replacement therapy (KRT) ${ }^{9-12}$ Outpatient KRT in the form of intermittent haemodialysis (iHD) and peritoneal dialysis (PD) has been linked to poor neurocognitive outcomes due to vascular and metabolic disturbances, ${ }^{13}$ and adults initiated on dialysis in the ICU may be particularly at risk due to their superimposed critical illness.

The goal of this study is to examine the relationship between KRT, delirium and long-term structural and cognitive outcomes in critically ill patients. We urgently need the results of such studies to reveal risk factors for poor cognitive and neurological outcomes in patients treated with KRT after ICU discharge, and to inform how different KRT modalities affect cognitive function in these vulnerable patients.

\section{Overall hypothesis}

We hypothesise that KRT is associated with low cerebral oxygenation, which in turn will be associated with delirium and long-term structural and functional neurological consequences at 3 and 12 months. We further hypothesise that iHD is a risk factor for lower cerebral oxygenation relative to continuous KRT (CKRT), due to rapid haemodynamic shifts. These impairments will have negative effects on patient quality of life.

\section{KRT in the ICU}

The decision to initiate KRT in patients admitted to the ICU is largely driven by medically refractory fluid overload, uraemia, electrolyte disturbances or metabolic acidosis, often in the setting of severe AKI. ${ }^{14}$ The burden of dialysis-requiring AKI is rising, ${ }^{15} 16$ particularly among critically ill adults. ${ }^{15}$ Over half of all adults admitted to the ICU will develop AKI,${ }^{11}$ with $3 \%-13 \%$ ultimately requiring treatment with KRT. ${ }^{12}$ The risk of acquiring AKI among hospitalised or critically ill patients further increases with age and the presence of other comorbid conditions including chronic kidney disease, heart disease, hypertension, diabetes, dementia and cancer. ${ }^{17}$

Three modalities of KRT are available for use in the acute setting: iHD, CKRT, and PD, with the former two being the most frequently used in patients admitted to the ICU. ${ }^{18}$ iHD provides the most efficient clearance, but is poorly tolerated in haemodynamically unstable patients (eg, those requiring haemodynamic support with vasopressors) ${ }^{19}$ CKRT uses slower rates of blood and dialysate flow over longer time periods, and is consequently more amenable to use in unstable populations..$^{20}$ Many patients started on CKRT are often later transitioned to iHD as they become more capable of tolerating rapid haemodynamic shifts. However, unlike CKRT in which the brain's ability to regulate blood flow in response to changing blood pressures (cerebral autoregulation) appears to be unperturbed, ${ }^{21}$ the rapid shifts during iHD are known to affect cerebral perfusion. ${ }^{22}$ No studies to date have explored the implications of dialysis or of transitioning from CKRT to iHD on cerebral perfusion.

Regional cerebral oxygen saturation as a surrogate marker of cerebral perfusion during critical illness and KRT

Regional cerebral oxygen saturation ( $\mathrm{rSO} 2)$ in the frontal cortex-a surrogate marker of overall brain oxygenation-is a reliable marker of cerebral perfusion ${ }^{23}$ and can be used to evaluate cerebral autoregulation. ${ }^{24} \mathrm{rSO} 2$ can be measured in critically ill patients using NIRS, in which a light sensor placed on the patient's forehead is used to non-invasively assess the oxygenation level of haemoglobin in the underlying brain tissue. ${ }^{25}$ Cerebral blood flow and rSO2 are reduced in adults with endstage kidney disease undergoing long-term maintenance dialysis,${ }^{26}$ and have been associated with poor cognitive outcomes. ${ }^{27-29}$ Moreover, low rSO2 in other populations has been correlated with neurological abnormalities on brain imaging. ${ }^{30}$ Still, longitudinal studies examining the consequences of short-term acute dialysis on $\mathrm{rSO} 2$ and its relationship with long-term cognitive function and neuroimaging have yet to be undertaken.

Rapid changes in mean arterial pressure (MAP) during iHD are generally well tolerated among stable individuals in the ICU, and providers have traditionally operated under the assumption that cerebral autoregulation is unperturbed by these rapid haemodynamic shifts. However, recent data from our group have demonstrated that cerebral autoregulation is disturbed in critically ill patients not undergoing dialysis. ${ }^{31}$ An understanding of the consequences of dialysis on cerebral autoregulation in critically ill patients is needed to guide decision-making regarding choice of KRT modality in the ICU.

\section{Neurocognitive outcomes of AKI}

AKI is associated with an increased risk for long-term neurological issues, including inflammation, stroke, delirium and cognitive deficits, including dementia. ${ }^{32-35}$ Prior research by our group has further demonstrated that survivors of AKI experience quantifiable deficits in the areas of attention, visuomotor and executive function. ${ }^{36}$

\section{Neurocognitive outcomes of KRT}

Maintenance dialysis is associated with short- and longterm neurocognitive impairment, including a decline in executive function. ${ }^{37-40} \mathrm{Up}$ to $70 \%$ of patients undergoing maintenance iHD demonstrate cognitive impairment. ${ }^{41}$ Studies in patients with chronic kidney disease (CKD) have posited that for every $10 \mathrm{~mL} / \mathrm{min} / 1.73 \mathrm{~m}^{2}$ decline in estimated glomerular filtration rate (eGFR) below $60 \mathrm{~mL} / \mathrm{min} / 1.73 \mathrm{~m}^{2}$, an $11 \%$ increased prevalence of cognitive dysfunction occurs. ${ }^{42}$ This decline is further pronounced in individuals with CKD undergoing 
treatment with dialysis, due to their increased risk for cerebrovascular disease. ${ }^{27} 43$ Despite this understanding of the cognitive outcomes of kidney dysfunction and KRT in the chronic setting, the neurocognitive impact of acute KRT remains largely unknown, and cognition has seldom been the focus of KRT trials in the ICU.

Patients on iHD may be particularly vulnerable to adverse neurocognitive effects, as a result of the rapid haemodynamic shifts that occur during treatment. To this end, cognitive fluctuations have been noted as a consequence of iHD, including deterioration in cognitive function post-iHD compared with pre-iHD levels, particularly in areas of attention and executive function. ${ }^{44}$

\section{Kidney disease and critical illness independently predict long-} term structural brain pathology

Adults with CKD exhibit structural neurological changes on neuroimaging. eGFR in healthy controls has been inversely associated with white matter microstructural integrity in a diffusion tensor imaging study of the brain, as evidenced by lower fractional anisotropy (FA) and higher mean diffusivity (MD) in those with lower eGFR. ${ }^{45}$ Furthermore, CKD has been linked to global brain atrophy, demonstrated by increased lateral ventricle and sulci dilation, ${ }^{46}$ and reduced white matter volume. ${ }^{47}$

Additional neuroimaging findings have been found in those with CKD on dialysis. White matter hyperintensities are more prevalent in patients on iHD relative to their healthy counterparts, ${ }^{48}$ and those on iHD have an increased ventricular-brain ratio relative to healthy controls. ${ }^{49}$

ICU survivors show a similar increase in ventriclebrain ratio suggestive of cerebral atrophy compared with age-matched controls. ${ }^{50}$ In the same study, the authors found a correlation between duration of delirium and degree of frontal and temporal lobe atrophy ${ }^{50}$ It is not clear whether any patients in that study had CKD, AKI or required CKRT.

\section{Study aims and hypotheses}

\section{Impact of dialysis on rSO2 and delirium}

Patients on maintenance dialysis experience an intradialytic reduction in cerebral oxygenation. ${ }^{23}$ Reduced cerebral oxygenation in other contexts in the ICU is known to be associated with delirium. ${ }^{7}$ The effect of dialysis on $\mathrm{rSO} 2$, and the effect of intradialytic $\mathrm{rSO} 2$ on delirium in the ICU is unknown. We hypothesise that patients undergoing KRT will demonstrate an intradialytic drop in $\mathrm{rSO} 2$ from baseline, and that more pronounced reductions in $\mathrm{rSO} 2$ will be associated with increased severity of delirium. We further hypothesise that lower intradialytic rSO2, longer durations of disturbed cerebral autoregulation and larger areas under the curve (AUC) outside of patient's optimal MAP will be associated with increased severity of delirium.
Long-term consequences of intradialytic rSO2 at 3 and 12 months post-ICU discharge

The long-term impact of alterations in cerebral oxygenation during acute dialysis is unknown. We hypothesise that intradialytic rSO2 during acute dialysis will be correlated with reduced neurocognitive function at 3 and 12 months after ICU discharge, and that patients with worse delirium scores will exhibit greater cognitive dysfunction. We further hypothesise that cognitive impairment at 3 and 12 months will be associated with an increased risk for poorer adherence to treatment regimens, adverse events and increased rate of driving accidents. ${ }^{51}$

Poor kidney function is associated with structural neurological impairment in patients with CKD and on maintenance dialysis. Low cerebral oxygenation and delirium are independently correlated with neurological abnormalities on imaging. The effect of dialysis and rSO2 during critical illness on structural neuroanatomy is unknown. We hypothesise that severe, prolonged delirium and lower rSO2 during dialysis in the ICU are associated with structural neurological impairment at a macrostructural and microstructural level.

\section{Differential effects of different dialysis modalities on rSO2}

Patients on maintenance dialysis exhibit lower rSO2 during dialysis sessions ${ }^{23}$; the impact of short-term dialysis on rSO2 is unknown. We hypothesise that iHD is a risk factor for lower rSO2 versus CKRT, due to rapid haemodynamic shifts.

\section{Hypothesised causal pathway}

The hypothesised causal pathway is outlined in figure 1. AKI resulting in the receipt of KRT will lead to a reduction in cerebral oxygenation from baseline. This reduced cerebral oxygenation will be associated with an increased risk for delirium. Downstream consequences of low cerebral oxygenation and delirium will include reduced brain structural integrity (as evidenced by high MD and low FA), increased brain atrophy, reduced cognitive function, and reduced patient well-being (evidenced by a lower ability to adhere to prescribed medication regimens and reduced driving safety).

\section{METHODS AND ANALYSIS}

\section{Patient and public involvement}

Patient representatives who have experienced critical illness will be sought prior to study initiation, to inform both patient-centred long-term outcome data collection as well as knowledge dissemination.

\section{Study design and setting}

This is a prospective, observational cohort study of critically ill participants initiated on acute KRT (iHD or CKRT). The Identifying neurocognitive outcomes and cerebral oxygenation in critically ill adults on acute kidney replacement therapy in the intensive care unit 


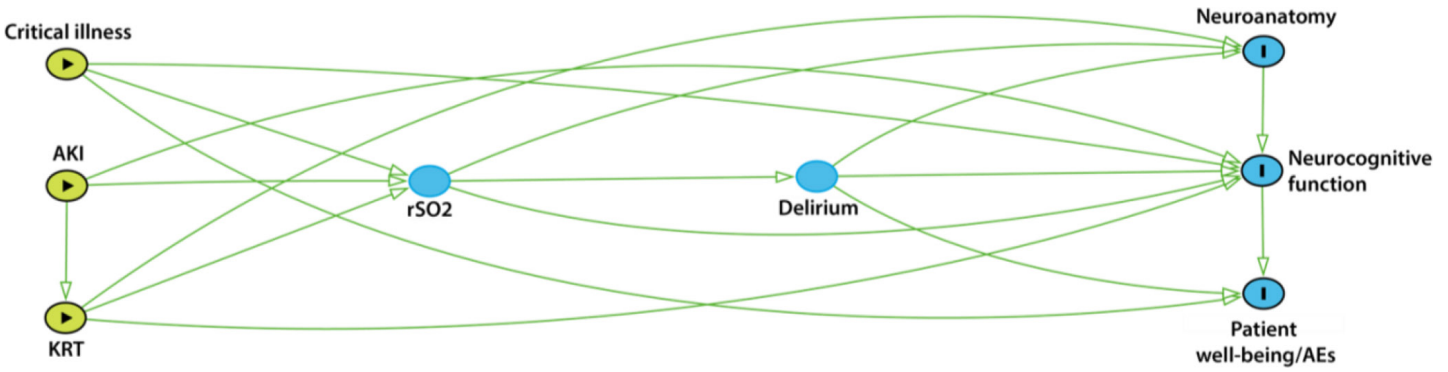

Figure 1 Hypothesised causal pathway. AE, adverse events; AKI, acute kidney injury; KRT, kidney replacement therapy; rSO2, regional cerebral oxygen saturation. Neurocognitive function is defined as performance on the Repeatable Battery for the Assessment of Neuropsychological Status (RBANS) and Kinarm tasks.

(INCOGNITO-AKI) study will take place in a 33-bed medical-surgical ICU at a tertiary academic hospital in Ontario, Canada.

\section{Eligibility criteria}

The inclusion criteria for the INCOGNITO-AKI study are: age greater than or equal to 18 years; admitted to the Kingston Health Sciences Centre (KHSC) ICU; diagnosis of severe AKI requiring KRT (defined by the presence of either a twofold increase in serum creatinine from baseline, serum creatinine level greater than or equal to 354 $\mu \mathrm{mol} / \mathrm{L}$ with an increase of $27 \mu \mathrm{mol} / \mathrm{L}$ from baseline or urine output $<6 \mathrm{~mL} / \mathrm{kg}$ in the preceding 12 hours $^{52}$ ); and within 12 hours of initiation of KRT via iHD or CKRT.

Patients will be excluded from the study if they have acquired or congenital neurological disorders; any contraindication to testing with cerebral oximetry, Kinarm or MRI (eg, claustrophobia, limb amputation, paresis, neuromuscular disorders); KRT via PD; failure to consent; life expectancy less than 24 hours; clinical suspicion of renal obstruction, rapidly progressive glomerulonephritis or interstitial nephritis, or prehospitalisation $\mathrm{eGFR}<30 \mathrm{~mL} / \mathrm{min} / 1.73 \mathrm{~m}^{2}$.

\section{Recruitment and consent}

Patients will be screened for eligibility and recruited by the research team from the KHSC ICU within 12 hours of initation of KRT. Patients will be evaluated for capacity to consent on an ongoing basis. If unable to consent individually, consent will be obtained from the participant's substitute decision-maker.

Participants will be informed of their right to withdraw from the study at any time. Should a participant feel claustrophobic within the MRI or Kinarm robotic device during any of the assessments, or experience any other perceived or real adverse symptoms, the assessment will be stopped immediately, and the participant will be given the option of reattempting the assessment at a later date or withdrawing from the study altogether.

To facilitate recruitment and retention, compensation for time and travel will be provided. Follow-up visits will be scheduled when patients are already returning to KHSC for clinical care, and will be offered at flexible times. Recruitment materials will represent the diverse spectrum of age, sex and gender within the critical care population.

On consent, participants will undergo a variety of assessments in accordance with the INCOGNITO-AKI Study Schema (figure 2). Assessments will be obtained purely for investigational purposes and will not alter the patient's treatment in any way.

\section{Data collection}

Patients admitted to the KHSC ICU receiving KRT will be enrolled within 12 hours of KRT initiation (ideally within 6 hours). Reasons for exclusion of screened but ineligible participants will be recorded. Baseline data including demographic information (age, sex, gender, ethnicity, geographical location (first 3 digits of postal code)), medications (including antihypertensive medications), comorbidities (including hypertension and history of mental illness eg, depression, anxiety), reason for ICU admission, frailty (assessed by Clinical Frailty Scale, CFS), baseline cognition (Clinical Dementia Rating Scale, CDRS) and illness severity (assessed via Acute Physiological Assessment and Chronic Health Evaluation, APACHE II) will be collected from patient's medical records and via self-report.

Delirium will be assessed daily during ICU admission using the Confusion Assessment Method (CAM)-ICU-7 delirium severity scale ${ }^{53}$ which requires daily assessment using the Richmond Agitation Sedation Scale (RASS) to quantify patients' level of sedation/consciousness. Additional ICU-specific data elements will be collected, including indication for dialysis, type of KRT (iHD vs CKRT, if CKRT then continuous veno-venous hemofiltration vs continuous veno-venous hemodialysis vs continuous veno-venous hemodiafiltration), indication for type of KRT, kidney function, dialysis-specific variables (dialysate/replacement fluid rates, electrolytes, access, blood flow, rate of fluid removal, hypertensive and hypotensive episodes, duration of treatment, type of anticoagulation), duration of KRT and ICU length-of-stay.

At time of hospital discharge, hospital length-of-stay, discharge destination, kidney function (eGFR), and ongoing maintenance dialysis requirements will be recorded. 


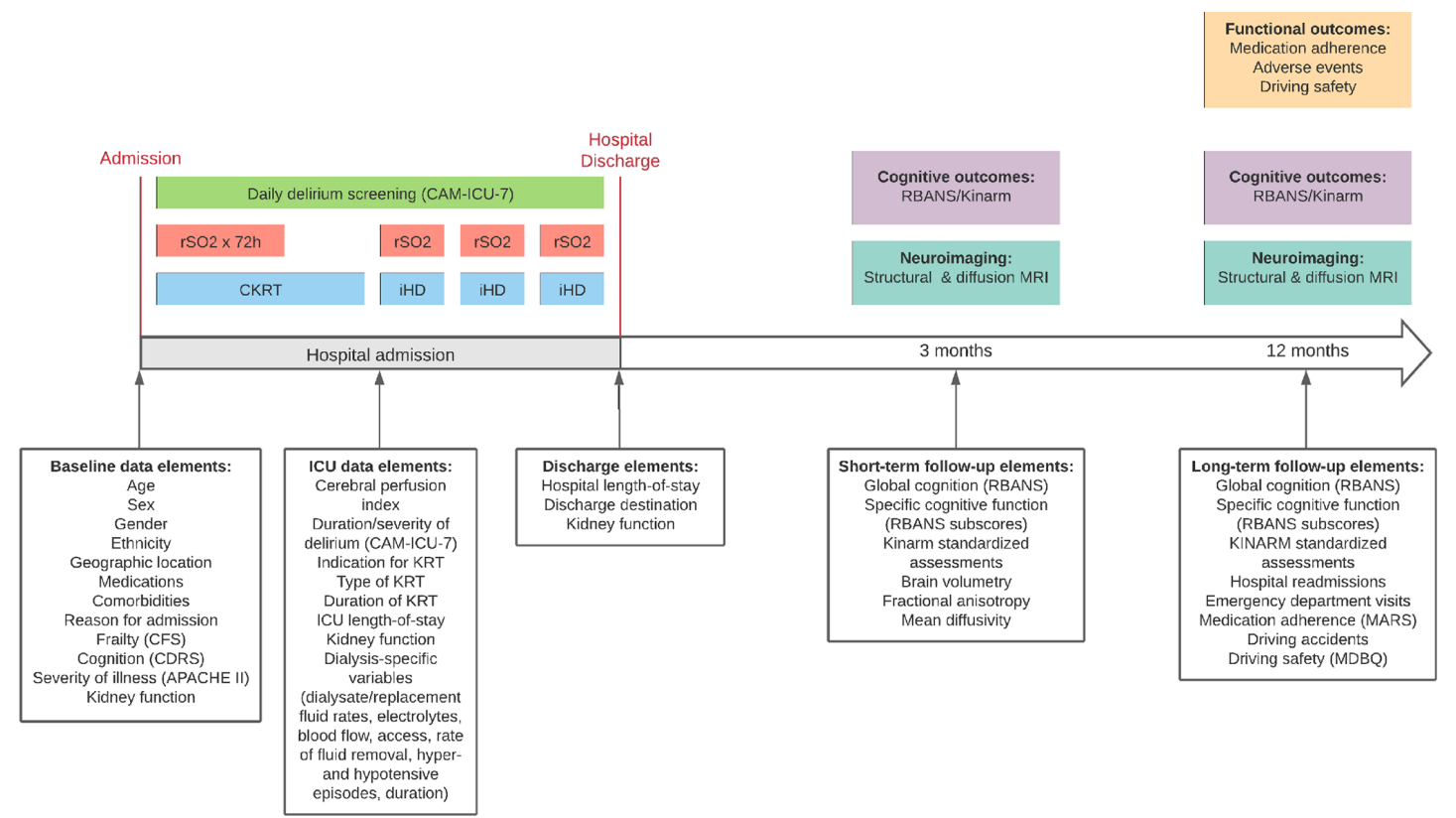

Figure 2 Study schema. APACHE II, Acute Physiological Assessment and Chronic Health Evaluation; CAM-ICU, Confusion Assessment Method-Intensive Care Unit; CDRS, Clinical Dementia Rating Scale; CFS, Clinical Frailty Scale; CKRT, continuous $\mathrm{KRT}$; iHD, intermittent haemodialysis; KRT, kidney replacement therapy; RBANS, Repeatable Battery for the Assessment of Neuropsychological Status; rSO2, regional cerebral oxygen saturation; MARS, Medication Adherence Rating Scale; MDBQ, Machester Driver Behavior Questionnaire.

During participants' 3 and 12 month follow-up visits, information on their maintenance dialysis requirements, as well as their most recent laboratory data for creatinine/ eGFR, will be recorded.

\section{Outcomes}

Cerebral oximetry

Cerebral oxygenation can be continuously monitored with non-invasive oximeters employing NIRS to generate rSO2 values. ${ }^{54}$ Participants on CKRT will undergo continuous cerebral oximetry using the CASMED FORESIGHT Elite cerebral oximeter (Edwards LifeSciences, USA) beginning 1 hour prior to, and during the first 72 hours of CKRT. Post-CKRT oximetry will be measured for 1 hour following completion of CKRT. Participants on iHD will undergo oximetry beginning 1 hour prior to each iHD session, continuously throughout each session and ending 1 hour following completion of each session.

\section{Neurocognitive testing}

\section{Kinarm}

Kinarm End-Point Lab (Kinarm, Kingston, Canada) is an interactive robotic technology that uses a battery of behavioural tasks, called Kinarm Standard Tests (KST), that precisely quantifies sensory, motor and neurocognitive impairment. ${ }^{55}$ Our group has previously demonstrated the feasibility of using the Kinarm for neurocognitive assessment in ICU survivors. ${ }^{56}$ Participants will undergo serial Kinarm assessment at 3 and 12 months after discharge. Reasons for non-completion of Kinarm assessments will be recorded.

The specific Kinarm tasks to be used in the INCOGNITO-AKI study are outlined in table 1.
All Kinarm task-specific parameters will be standardised to available normative control data generated as part of the validation process for the Kinarm robot, accounting for age, sex, handedness and Kinarm platform effects. ${ }^{57}$ Task-specific parameter values are then summed to generate global task scores for each participant, providing an assessment of overall performance on a given task. All scores will be normalised to z-scores. Neurocognitive impairment in the INCOGNITO-AKI study will be defined as a task $z$-score greater than the 95 th percentile (ie, z-scores greater than 1.64 indicate impairment on the given task relative to the healthy control cohort used to validate $\mathrm{KST}$ ), in accordance with previously published literature using the Kinarm robot. ${ }^{58}$

\section{Repeatable battery for the assessment of neuropsychological Status}

Global cognition will be assessed using the Repeatable Battery for the Assessment of Neuropsychological Status (RBANS) at the same time points. The RBANS is a $30 \mathrm{~min}$ tool used to screen and quantify cognitive impairment in adults, across a variety of domains ${ }^{59}$ and is commonly used to assess cognition in ICU survivors. Neurocognitive domains assessed through the RBANS include immediate and delayed memory, visuospatial/constructional, language and attention. ${ }^{59}$

\section{Functional outcomes}

Functional patient outcomes will be assessed at 12 months after discharge, and will include assessment of medication adherence using the Medication Adherence Rating Scale (MARS); assessment of adverse events including number of rehospitalisations and emergency department 
Table 1 Kinarm tasks and domains of neurocognitive function assessed in the INCOGNITO-AKI study

\begin{tabular}{lll}
\hline Task & Task type & Neurocognitive domain \\
\hline Arm position matching & Sensorimotor & Somatosensory processing for perception, position-sense \\
Ball on bar & Sensorimotor & Bi-manual coordination, visuomotor skills \\
$\begin{array}{l}\text { Visually guided reaching } \\
\text { Reverse visually guided } \\
\text { reaching }\end{array}$ & Sensorimotor & Motor coordination, visuomotor skills, postural control of arm \\
Object hit & Cognitive-motor & Rapid visuomotor skills, bi-manual motor planning, spatial attention \\
Object hit and avoid & Cognitive-motor & $\begin{array}{l}\text { Rapid motor decisions, bi-manual motor planning, spatial attention, } \\
\text { executive function (attention and inhibitory control) }\end{array}$ \\
\hline Trails A\&B & Sensory-cognitive & Executive function, task switching \\
Paired associate learning & Sensory-cognitive & Visuospatial working memory \\
\hline
\end{tabular}

Adapted from the Kinarm user guide. ${ }^{57}$

INCOGNITO-AKI, Identifying neurocognitive outcomes and cerebral oxygenation in critically ill adults on acute kidney replacement therapy in the intensive care unit.

visits; and driving safety (number of motor vehicle accidents and Manchester Driver Behaviour Questionnaire $(\mathrm{MDBQ})$ ).

\section{Structural neuroimaging}

Patients will undergo serial structural MRI of the brain at 3 and 12 months after discharge. Anonymised and deidentified MRI scans will be processed using Medical Image Processing, Analysis, and Visualization (MIPAV) ${ }^{60}$ V.7.3.0 and Oxford Centre for Functional MRI of the Brain Software Library $(\mathrm{FSL})^{61}$ V.5.0 medical image processing programmes. Scans will be corrected for intensity nonuniformity $^{62}$ and transformed into a common image space to adjust for variations in head size and orientation. ${ }^{63}$ Skull stripping will be performed. ${ }^{64}$ Automated and semiautomated techniques will be used to determine whole-brain volumes from T1-weighted images ${ }^{64} 65$ for analysis of macrostructural brain integrity, as well as FA and MD measures from diffusion-weighted images ${ }^{63}$ for analysis of microstructural brain integrity. Brain volumes will be corrected for total intracranial volume to account for head size ${ }^{66}$ Reasons for non-completion of MRI scanning will be recorded. Patients will be used as their own controls over time using a within-subjects design.

\section{Sample size}

The relationship between cerebral oxygenation during dialysis and delirium in critically ill patients has not been previously explored. However, previous research by our group has demonstrated a correlation of 0.34 between cerebral oximetry parameters (ie, dysfunctional cerebral autoregulation) and delirium in critically ill adults. ${ }^{31}$ Assuming a similar correlation will be found through the present study, then 93 patients would be needed to detect a correlation of 0.34 at a two-sided 0.05 significance level with $90 \%$ power. Given that we do not know what the correlation in critically ill patients on KRT will be, we have based our effect size estimate and consequently our sample size on what is clinically relevant for this population, as well as on estimated enrolment rates. One hundred and eight subjects will provide $90 \%$ power to detect a correlation of 0.3 between two variables, and $90 \%$ power to detect a predictor that explains $9 \%$ of the remaining variance in a dependent variable of a linear regression model after controlling for other predictors in the model. Given that delirium and long-term neurocognitive trajectories are multifactorial, and cerebral oxygenation during the first 72 hours of CKRT or during iHD is likely to be a small but highly relevant component of this, these detection limits are clinically meaningful in this population. Approximately five new adult patients are initiated on KRT in the ICU per month at KHSC. Assuming a $90 \%$ consent rate based on our prior work, 54 patients per year will be enrolled in the INCOGNITO-AKI study over a 2-year period, yielding an estimated sample size of 108 patients.

\section{Data management}

All information collected from patients will be recorded directly into a Research Electronic Data Capture database housed on the Queen's University server, which can only be accessed by study team members (https:// fhs.cac.queensu.ca/CNS/). All patient identifiers will be coded and anonymised. All results from Kinarm and NIRS assessments will be assigned a numeric code associated with each subject and otherwise stripped of all identifying information, and with access restricted to study personnel only. A master linking log connecting patient's identifiable information (ie, medical record number) to their study ID will be securely maintained internally behind two locks, with access restricted to study personnel. MRI scans consisting of a series of digital image communication (DICOM) files will be assigned a numeric code associated with each subject and otherwise stripped of all identifying information using a standard DICOM file anonymiser. 


\section{Statistical methods}

Primary objectives

The primary objective of this study is the association between intradialytic $\mathrm{rSO} 2$ and delirium during ICU admission, as measured by NIRS. Continuous rSO2 data throughout each form of dialysis will be summarised using four variables, which together comprise the Cerebral Perfusion Index (CPI): (1) mean intradialytic rSO2, (2) delta-rSO2, (3) duration of disturbed cerebral autoregulation and (4) AUC outside of the optimal MAP. ${ }^{67}$ These four summary variables have been used to describe cerebral oxygenation in previous reports. ${ }^{67-69}$

Mean intradialytic rSO2 will be calculated for the duration of oximetry recording during the first CKRT session (72 hours during CKRT, as described above), and for each iHD session recording period. Mean intradialytic rSO2 will be calculated as the mean $\mathrm{rSO} 2$ of all total KRT minutes, irrespective of whether the patient has been initiated on CKRT, iHD, or a combination thereof.

Delta-rSO2 will be calculated as the absolute difference between the mean intradialytic $\mathrm{rSO} 2$ (as described above) and the mean baseline $\mathrm{rSO} 2$, where mean baseline $\mathrm{rSO} 2$ is defined as the mean $\mathrm{rSO} 2$ during the 1 hour oximetry recording preinitiation of CKRT, or the mean rSO2 during the 1-hour oximetry recording preinitiation of each iHD session.

Cerebral autoregulation will be evaluated using the cerebral oximetry index (COx), with time-varying Spearman correlation coefficients calculated between rSO2 values and MAP at 1 min intervals throughout the duration of the oximetry recordings. ${ }^{31}$ Positive values of the COx $(p<0.0001)$ are indicative of disturbed cerebral autoregulation. ${ }^{31}$ Cumulative duration of disturbed cerebral autoregulation will be taken as the length of time during each recording session for which COx values were positive. ${ }^{67} 68$ Duration of disturbed cerebral autoregulation will be taken as the percentage of total recording time during which cerebral autoregulation was disturbed (ie, the cumulative duration of cerebral autoregulation dysfunction will be divided by the total recording time and then multiplied by 100 to calculate the percentage of time with cerebral autoregulation dysfunction). ${ }^{31}$

Optimal MAP \pm SD will be calculated as the mean MAP for COx of $0 \pm 1 \mathrm{SD} .{ }^{67}$ AUC outside of the optimal MAP will be taken as the proportion of the AUC where AUC was outside of the range of the optimal MAP. ${ }^{67}$

Multiple linear regression using the four CPI parameters as the independent predictor variables and cumulative CAM-ICU-7 score throughout the patient's ICU stay as the response variable will be performed. The model will be built from the following potential covariates: age, illness severity APACHE score, baseline cognition via CDRS, and baseline frailty via CFS.

\section{Secondary objectives}

To assess the relationship between rSO2, delirium and long-term cognitive impairment, multiple linear regression will be used to determine the association between
CPI parameters (predictor variables) and neurocognitive impairment via RBANS/Kinarm scores at 3 and 12 months (response variables), controlling for covariates (age, frailty, dementia, illness severity, delirium).

Association between $\mathrm{rSO} 2$ values (predictor) and each of brain volume, FA and MD (outcomes) will be assessed through multiple linear regression. Baseline CDRS score, APACHE score, and CFS will be included as covariates. Linear regression will be used to determine the association between structural brain pathology (total brain volume, FA and $\mathrm{MD}$ ) and neurocognitive function (RBANS/Kinarm scores), as well as patient well-being (adverse events, MARS and MDBQ scores) at 3 and 12 months post-discharge.

\section{Tertiary/exploratory objectives}

Our exploratory objective is to assess the differential effect of iHD and CKRT on cerebral oxygenation. To this end, analysis of covariance will be used to assess effect of first dialysis modality (iHD vs CKRT) on delta-rSO2 values, while controlling for covariates including baseline haemodynamic status and age. Baseline haemodynamic status will be assessed using information from the APACHE II illness severity scale (ie, vitals) and RASS score at baseline to evaluate consciousness level. Delta-rSO2 values will be used to determine differential changes from baseline throughout iHD and CKRT.

\section{Ethics and dissemination}

This study has been approved by the Queen's University Health Sciences and Affiliated Teaching Hospitals Research Ethics Board (HSREB), REB approval number: DMED-2424-20. The study protocol does not interfere in any way with the standard of care provided to patients.

\section{Risks of participation}

Use of the Kinarm and NIRS are low-risk. There is a risk of incidental findings discovered by Kinarm. In this event, participants will be referred to a neurologist for further examination. Results will be communicated to the participant and/or family via a brief written research report. There is a risk of skin irritation due to the adhesive used in the NIRS device.

MRI is low risk. No contrast agents (eg, gadolinium) will be used due to the risk of nephrogenic systemic fibrosis in patients with kidney impairment. There is a risk of incidental findings discovered by MRI. In this event, participants will be referred to a neurologist for further examination. Results will be communicated to the participant and/or family via a brief written research report.

In spite of our best efforts, if personal health information is inappropriately released, we will take measures to ensure that further release of information is stopped, that any information which can be retrieved is retrieved immediately, and that the KHSC and Queen's University Privacy Office and REB will be notified. Further actions may be taken according to recommendations from the privacy office and REB. 


\section{Knowledge translation}

Prior to study initiation, functional patient-centred outcomes will be sought from diverse patient representatives who have experienced critical illness, and included in the follow-up data collection for the INCOGNITO-AKI study to elucidate meaningful consequences of poor cognition. To inform knowledge translation, these representatives will also be asked for input on how to disseminate and increase accessibility of study results (eg, multiple languages, print and electronic dissemination, use of text and graphics to represent data). Results of the INCOGNITO-AKI study will be presented at critical care conferences, and disseminated to ICU patients and families in their preferred method.

\section{Significance}

Initiation of acute KRT is increasingly common in critically ill adults admitted to the ICU. This project will provide crucial insight into the early neurological changes occurring in these patients and their short-term and long-term impact on cognitive function. If we discover that cerebral oxygenation is lower when patients are on iHD versus when they are on CKRT and that this reduction is associated with poorer cognition and neuroimaging findings, this will provide a rationale for developing a protocol to maximise $\mathrm{rSO} 2$ and control ultrafiltration rate during iHD. This understanding will further serve as a foundation for developing interventions to improve neurological outcomes in this vulnerable cohort, thereby reducing their overall morbidity and mortality and relieving stress on an already burdened healthcare system.

\footnotetext{
Author affiliations

${ }^{1}$ Centre for Neuroscience Studies, Queen's University, Kingston, Ontario, Canada

${ }^{2}$ Department of Medicine, Queen's University, Kingston, Ontario, Canada

${ }^{3}$ Department of Biomedical and Molecular Sciences, Queen's University, Kingston,

Ontario, Canada

${ }^{4}$ Department of Public Health Sciences, Queen's University, Kingston, Ontario,

Canada

${ }^{5}$ Kingston General Health Research Institute, Kingston, Ontario, Canada

${ }^{6}$ Department of Diagnostic Radiology, Queen's University, Kingston, Ontario, Canada

${ }^{7}$ Critical Care Medicine, Queen's University, Kingston, Ontario, Canada

${ }^{8}$ Department of Critical Care Medicine, Kingston General Hospital, Kingston, Ontario, Canada
}

Twitter Natasha Arianne Jawa @JawaTasha, Samuel A Silver @drsamsilver, Stephen H Scott @ScottLIMBlab, David M Maslove @DavidMaslove, John Muscedere @muscedej and John Gordon Boyd @jgordonboyd

Contributors JGB and NAJ designed the study and wrote the study protocol. NAJ drafted and revised the manuscript. JGB, RMH, SAS, BYMK, PAN, AGD, DMM, $\mathrm{JM}$, and SHS reviewed and revised the manuscript. SHS developed, designed and manages the Kinarm robotic labs that will be used in the INCOGNITO-AKI study.

Funding The authors have not declared a specific grant for this research from any funding agency in the public, commercial or not-for-profit sectors.

Competing interests SHS is cofounder and CSO of Kinarm that commercialises the Kinarm robotic technology used in the present study. NAJ, RMH, SAS, AGD, PAN, BYMK, DMM, JM and JGB have no conflicts of interest to declare.

Patient and public involvement Patients and/or the public were not involved in the design, or conduct, or reporting, or dissemination plans of this research.

Patient consent for publication Not required.

Provenance and peer review Not commissioned; externally peer reviewed.
Open access This is an open access article distributed in accordance with the Creative Commons Attribution Non Commercial (CC BY-NC 4.0) license, which permits others to distribute, remix, adapt, build upon this work non-commercially, and license their derivative works on different terms, provided the original work is properly cited, appropriate credit is given, any changes made indicated, and the use is non-commercial. See: http://creativecommons.org/licenses/by-nc/4.0/.

ORCID iD

Natasha Arianne Jawa http://orcid.org/0000-0001-7600-7178

\section{REFERENCES}

1 Rawal G, Yadav S, Kumar R. Post-intensive care syndrome: an overview. J Trans/ Int Med 2017;5:90-2.

2 Pandharipande PP, Girard TD, Jackson JC, et al. Long-term cognitive impairment after critical illness. N Engl J Med 2013;369:1306-16.

3 Quasim T, Brown J, Kinsella J. Employment, social dependency and return to work after intensive care. J Intensive Care Soc 2015;16:31-6.

4 Kamdar BB, Suri R, Suchyta MR, et al. Return to work after critical illness: a systematic review and meta-analysis. Thorax 2020;75:17-27.

5 Girard TD, Jackson JC, Pandharipande PP, et al. Delirium as a predictor of long-term cognitive impairment in survivors of critical illness. Crit Care Med 2010;38:1513-20.

6 Maldonado JR. Acute brain failure: pathophysiology, diagnosis, management, and sequelae of delirium. Crit Care Clin 2017;33:461-519.

7 , Wood MD, et al, Cerebral Oxygenation and Neurological Outcomes Following Critical Illness (CONFOCAL) Research Group, Canadian Critical Care Trials Group. Low brain tissue oxygenation contributes to the development of delirium in critically ill patients: a prospective observational study. J Crit Care 2017;41:289-95.

8 Siew ED, Fissell WH, Tripp CM, et al. Acute kidney injury as a risk factor for delirium and coma during critical illness. Am J Respir Crit Care Med 2017;195:1597-607.

9 Levey AS, Eckardt K-U, Dorman NM, et al. Nomenclature for kidney function and disease: report of a kidney disease: improving global outcomes (KDIGO) consensus conference. Kidney Int 2020;97:1117-29.

10 Wald R, McArthur E, Adhikari NKJ, et al. Changing incidence and outcomes following dialysis-requiring acute kidney injury among critically ill adults: a population-based cohort study. Am J Kidney Dis 2015;65:870-7.

11 Hoste EAJ, Bagshaw SM, Bellomo R, et al. Epidemiology of acute kidney injury in critically ill patients: the multinational AKI-EPI study. Intensive Care Med 2015;41:1411-23.

12 Uchino S, Kellum JA, Bellomo R, et al. Acute renal failure in critically ill patients: a multinational, multicenter study. JAMA 2005;294:813-8.

13 Seliger SL, Weiner DE. Cognitive impairment in dialysis patients: focus on the blood vessels? Am J Kidney Dis 2013;61:187-90.

14 Khwaja A. KDIGO clinical practice guidelines for acute kidney injury. Nephron Clin Pract 2012;120:c179-84.

15 Hsu RK, McCulloch CE, Dudley RA, et al. Temporal changes in incidence of dialysis-requiring AKI. J Am Soc Nephrol 2013;24:37-42.

16 Hsu RK, McCulloch CE, Heung M, et al. Exploring potential reasons for the temporal trend in dialysis-requiring AKI in the United States. Clin J Am Soc Nephrol 2016;11:14-20.

17 Wonnacott A, Meran S, Amphlett B, et al. Epidemiology and outcomes in community-acquired versus hospital-acquired AKI. Clin J Am Soc Nephrol 2014;9:1007-14.

18 Pannu N, Gibney RN. Renal replacement therapy in the intensive care unit. Ther Clin Risk Manag 2005;1:141-50.

19 Fathima N, Kashif T, Janapala RN, et al. Single-best choice between intermittent versus continuous renal replacement therapy: a review. Cureus 2019;11:e5558.

20 Chaves RCdeF, Tafner PFdoA, Chen FK, et al. Near-infrared spectroscopy parameters in patients undergoing continuous venovenous hemodiafiltration. Einstein 2019;17:eAO4439.

21 Schramm P, Closhen D, Wojciechowski J, et al. Cerebrovascular autoregulation in critically ill patients during continuous hemodialysis. Can J Anaesth 2013;60:564-9.

22 Polinder-Bos HA, García DV, Kuipers J, et al. Hemodialysis induces an acute decline in cerebral blood flow in elderly patients. J Am Soc Nephrol 2018;29:1317-25.

23 Polinder-Bos HA, Elting JWJ, Aries MJ, et al. Changes in cerebral oxygenation and cerebral blood flow during hemodialysis - a 
simultaneous near-infrared spectroscopy and positron emission tomography study. J Cereb Blood Flow Metab 2020;40:328-40.

24 Steiner LA, Pfister D, Strebel SP, et al. Near-infrared spectroscopy can monitor dynamic cerebral autoregulation in adults. Neurocrit Care 2009;10:122-8.

25 Ferrari M, Mottola L, Quaresima V. Principles, techniques, and limitations of near infrared spectroscopy. Can J Appl Physiol 2004;29:463-87.

26 Ito K, Ookawara S, Ueda Y, et al. Factors affecting cerebral oxygenation in hemodialysis patients: cerebral oxygenation associates with $\mathrm{pH}$, hemodialysis duration, serum albumin concentration, and diabetes mellitus. PLoS One 2015;10:e0117474.

27 Kovarova L, Valerianova A, Kmentova T, et al. Low cerebral oxygenation is associated with cognitive impairment in chronic hemodialysis patients. Nephron 2018;139:113-9.

28 Findlay MD, Dawson J, Dickie DA, et al. Investigating the relationship between cerebral blood flow and cognitive function in hemodialysis patients. J Am Soc Nephrol 2019;30:147-58.

29 Miyazawa $\mathrm{H}$, Ookawara S, Ito K, et al. Association of cerebral oxygenation with estimated glomerular filtration rate and cognitive function in chronic kidney disease patients without dialysis therapy. PLoS One 2018;13:e0199366.

30 Tian F, Morriss MC, Chalak L, et al. Impairment of cerebral autoregulation in pediatric extracorporeal membrane oxygenation associated with neuroimaging abnormalities. Neurophotonics 2017:4:041410.

31 Lee KF, Wood MD, Maslove DM, et al. Dysfunctional cerebral autoregulation is associated with delirium in critically ill adults. $J$ Cereb Blood Flow Metab 2019;39:2512-20.

32 Shiao C-C, Wu P-C, Huang T-M, et al. Long-term remote organ consequences following acute kidney injury. Crit Care 2015;19:438

33 Wu V-C, Wu P-C, Wu C-H, et al. The impact of acute kidney injury on the long-term risk of stroke. J Am Heart Assoc 2014;3. doi:10.1161/ JAHA.114.000933. [Epub ahead of print: 15 Jul 2014].

34 Liu M, Liang Y, Chigurupati S, et al. Acute kidney injury leads to inflammation and functional changes in the brain. J Am Soc Nephrol 2008;19:1360-70.

35 Baumgaertel MW, Kraemer M, Berlit P. Neurologic complications of acute and chronic renal disease. Handb Clin Neurol 2014;119:383-93.

36 Vanderlinden JA, Semrau JS, Silver SA, et al. Acute kidney injury is associated with subtle but quantifiable neurocognitive impairments. Nephrol Dial Transplant 2021. doi:10.1093/ndt/gfab161. [Epub ahead of print: 21 Apr 2021] (published Online First: 2021/04/22).

37 Iyasere $\mathrm{O}$, Brown EA. Cognitive function before and after dialysis initiation in adults with chronic kidney disease-a new perspective on an old problem? Kidney Int 2017;91:784-6.

38 Pereira AA, Weiner DE, Scott T, et al. Subcortical cognitive impairment in dialysis patients. Hemodial Int 2007;11:309-14.

39 Sarnak MJ, Tighiouart H, Scott TM, et al. Frequency of and risk factors for poor cognitive performance in hemodialysis patients. Neurology 2013;80:471-80.

40 Kalirao P, Pederson S, Foley RN, et al. Cognitive impairment in peritoneal dialysis patients. Am J Kidney Dis 2011;57:612-20.

41 Murray AM, Tupper DE, Knopman DS, et al. Cognitive impairment in hemodialysis patients is common. Neurology 2006;67:216-23.

42 Kurella Tamura M, Wadley V, Yaffe K, et al. Kidney function and cognitive impairment in US adults: the reasons for geographic and racial differences in stroke (regards) study. Am J Kidney Dis 2008;52:227-34.

43 Weiner DE, Scott TM, Giang LM, et al. Cardiovascular disease and cognitive function in maintenance hemodialysis patients. $A m \mathrm{~J}$ Kidney Dis 2011;58:773-81.

44 Costa AS, Tiffin-Richards FE, Holschbach B, et al. Clinical predictors of individual cognitive fluctuations in patients undergoing hemodialysis. Am J Kidney Dis 2014;64:434-42.

45 Sedaghat S, Cremers LGM, de Groot M, et al. Kidney function and microstructural integrity of brain white matter. Neurology 2015;85:154-61.

46 Rogova IV, Fomin VV, Damulin IV, et al. [Specific features of cognitive impairments in patients with predialysis chronic kidney disease]. Ter Arkh 2013;85:25-30.
47 Ikram MA, Vernooij MW, Hofman A, et al. Kidney function is related to cerebral small vessel disease. Stroke 2008;39:55-61.

48 Naganuma T, Takemoto Y, Shoji T, et al. Factors associated with cerebral white matter hyperintensities in haemodialysis patients. Nephrology 2012;17:561-8.

49 Yoshimitsu T, Hirakata H, Fujii K, et al. Cerebral ischemia as a causative mechanism for rapid progression of brain atrophy in chronic hemodialysis patients. Clin Nephrol 2000;53:445-51.

50 Gunther ML, Morandi A, Krauskopf E, et al. The association between brain volumes, delirium duration, and cognitive outcomes in intensive care unit survivors: the visions cohort magnetic resonance imaging study*. Crit Care Med 2012;40:2022-32.

51 Meyer J, Waldmann C, Driving WC. Driving (or not) after critical illness. J Intensive Care Soc 2015;16:186-8.

52 STARRT-AKI Investigators, Canadian Critical Care Trials Group, Australian and New Zealand Intensive Care Society Clinical Trials Group, et al. Timing of initiation of renal-replacement therapy in acute kidney injury. N Engl J Med 2020;383:240-51. doi:10.1056/ NEJMoa2000741

53 Khan BA, Perkins AJ, Gao S, et al. The confusion assessment method for the ICU-7 delirium severity scale: a novel delirium severity instrument for use in the ICU. Crit Care Med 2017;45:851-7.

54 Tosh W, Patteril M. Cerebral oximetry. BJA Educ 2016;16:417-21. doi:10.1093/bjaed/mkw024

55 Simmatis LER, Early S, Moore KD, et al. Statistical measures of motor, sensory and cognitive performance across repeated robotbased testing. J Neuroeng Rehabil 2020;17:86.

56 Wood MD, Maslove DM, Muscedere J, et al. Robotic technology provides objective and quantifiable metrics of neurocognitive functioning in survivors of critical illness:A feasibility study. J Crit Care 2018;48:228-36.

57 KST summary (analysis version 3.8) online, 2019. Available: https:// kinarm.com/support/user-guides-documentation/ [Accessed 05 Jan 2021].

58 Semrau JA, Herter TM, Scott SH, et al. Examining differences in patterns of sensory and motor recovery after stroke with robotics. Stroke 2015;46:3459-69.

59 Randolph C, Tierney MC, Mohr E, et al. The repeatable battery for the assessment of neuropsychological status (RBANS): preliminary clinical validity. J Clin Exp Neuropsychol 1998;20:310-9.

60 McAuliffe MJ, Lalonde FM, McGarry D. "Medical image processing, analysis and visualization in clinical research," proceedings 14th IEEE symposium on computer-based medical systems. CBMS 2001:381-6. doi:10.1109/CBMS.2001.941749

61 Woolrich MW, Jbabdi S, Patenaude B, et al. Bayesian analysis of neuroimaging data in fsl. Neuroimage 2009;45:S173-86.

62 Sled JG, Zijdenbos AP, Evans AC. A nonparametric method for automatic correction of intensity nonuniformity in MRI data. IEEE Trans Med Imaging 1998;17:87-97.

63 Andersson JL, Jenkinson M, Smith S. Non-linear registration a.k.a. spatial normalisation. In: FMRIB technical report. Oxford, United Kingdom: FMRIB Centre, 2007

64 Smith SM. Fast robust automated brain extraction. Hum Brain Mapp 2002;17:143-55.

65 Patenaude B, Smith SM, Kennedy DN, et al. A bayesian model of shape and appearance for subcortical brain segmentation. Neuroimage 2011;56:907-22.

66 Whitwell JL, Crum WR, Watt HC, et al. Normalization of cerebral volumes by use of intracranial volume: implications for longitudinal quantitative MR imaging. AJNR Am J Neuroradiol 2001;22:1483-9.

67 Khan JM, Wood MD, Lee KFH, et al. Delirium, cerebral perfusion, and high-frequency vital-sign monitoring in the critically ill. The CONFOCAL-2 feasibility study. Ann Am Thorac Soc 2021;18:112-21. doi:10.1513/AnnalsATS.202002-0930C

68 Wood MD, Khan J, Lee KFH, et al. Assessing the relationship between near-infrared spectroscopy-derived regional cerebral oxygenation and neurological dysfunction in critically ill adults: a prospective observational multicentre protocol, on behalf of the Canadian critical care trials group. BMJ Open 2019;9:e029189.

69 Semrau JS, Scott SH, Hamilton AG, et al. Quantified pre-operative neurological dysfunction predicts outcome after coronary artery bypass surgery. Aging Clin Exp Res 2020;32:289-97. 